Article

\title{
Characterization of Kink Actuators as Compared to Traditional Chevron Shaped Bent-Beam Electrothermal Actuators
}

\section{Ehab Rawashdeh *, Ayman Karam and Ian G. Foulds}

Physical Sciences and Engineering, Electromechanical Microsystems \& Polymer Integration Research (EMPIRe) Group, King Abdullah University of Science and Technology (KAUST), Thuwal 23955-6900, Saudi Arabia; E-Mails: ayman.karam@kaust.edu.sa (A.K.); ian.foulds@kaust.edu.sa (I.G.F.)

* Author to whom correspondence should be addressed; E-Mail: ehab.rawashdeh@kaust.edu.sa; Tel.: +966-553-422-604.

Received: 10 May 2012; in revised form: 19 June 2012 / Accepted: 3 July 2012 /

Published: 6 July 2012

\begin{abstract}
This paper compares the design and performance of kink actuators, a modified version of the bent-beam thermal actuator, to the standard chevron-shaped designs. A variety of kink and chevron actuator designs were fabricated from polysilicon. While the actuators were electrically probed, these designs were tested using a probe station connected to a National Instruments (NI) controller that uses LabVIEW to extract the displacement results via image processing. The displacement results were then used to validate the thermal-electric-structural simulations produced by COMSOL. These results, in turn, were used to extract the stiffness for both actuator types. The data extracted show that chevron actuators can have larger stiffness values with increasing offsets, but at the cost of lower amplification factors. In contrast, kink actuators showed a constant stiffness value equivalent to the chevron actuator with the highest amplification factor. The kink actuator also had larger amplification factors than chevrons at all designs tested. Therefore, kink actuators are capable of longer throws at lower power levels than the standard chevron designs.
\end{abstract}

Keywords: chevron; bent-beam; Kink; thermal actuators; simulation 


\section{Introduction}

This paper presents both experimental and simulation results in order to compare two designs of thermal actuators; The traditional bent-beam (chevron) actuator and the kink actuator (introduced in this paper). In order to produce displacement in a specific direction, a bent-beam thermal actuator uses its shape to amplify the thermal expansion of its beams. The heat required for actuation is usually generated by electrical resistive heating [1-3]. Electro-thermal actuators are capable of producing higher forces at lower voltage inputs than electrostatic actuators, however this comes at a cost of higher power consumption $[4,5]$. Thermal actuators have been used in various applications, such as 3D optical switching [6], micro-engines [7], on-chip nanomechanical testing [8], switching in RF MEMS [9], and various other applications [10,11]. Another bent-beam actuator that attempts to improve on the chevron actuator by making it also work as a linear force sensor is the z-shaped actuator [12]; however, kink and chevron actuators produce displacements which are an order of magnitude higher.

Chevron shaped actuators, a class of bent-beam thermal actuators shown in Figure 1(a), were first introduced by R. Cragun in 1999 [1], L. Que in 1999 [2] and M.J. Sinclair in 2000 [3]. A chevron's amplification factor (i.e., its total displacement) increases with decreasing bend angle (BA in Figure 2(a)). However, at very shallow angles, the standard chevron actuator's performance can become unpredictable from actuator to actuator due to process variations resulting from the accuracy of the masks used in the fabrication process.

Figure 1. A scanning electron microscope (SEM) image of (a) a Chevron actuator, and (b) a Kink actuator.

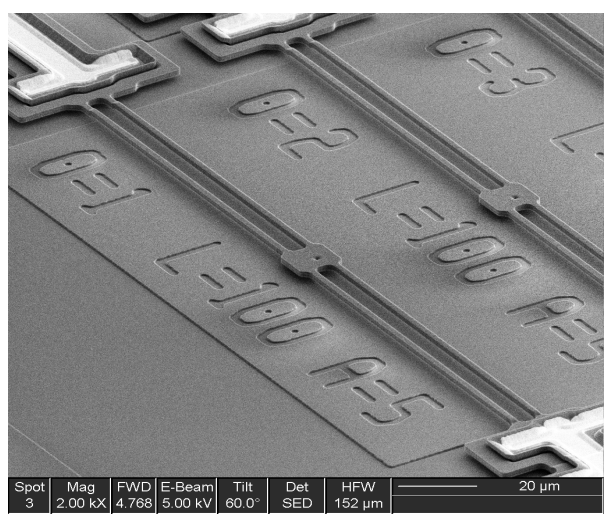

(a)

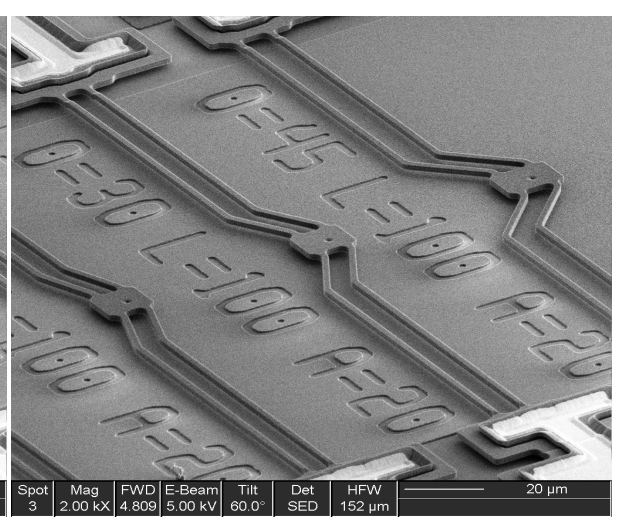

(b)

Figure 2. A schematic detailing the design parameters and the differences between (a) the Chevron and (b) the Kink actuator.

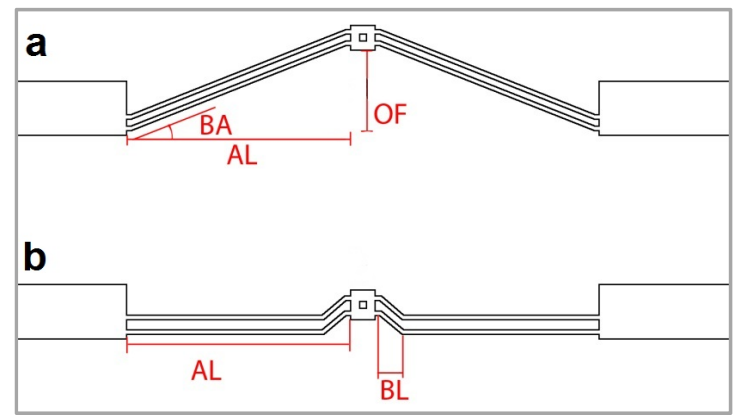


For this paper, actuators were fabricated using a mask resolution of $0.25 \mu \mathrm{m}$ [13], meaning that for a long beam with a 0.1 degree angle (the lowest angle tested), the rasterized mask consisted of a horizontal beam with a $0.25 \mu \mathrm{m}$ step every $143.20 \mu \mathrm{m}$. Our kink actuator was designed to improve the amplification factor; that is, for a given input power, it generates larger displacements than an equivalent chevron actuator.

The kink actuator, Figure 1(b), was designed to be a zero-angle chevron actuator with a small kink at the center in order to ensure buckling in the correct direction and reliable performance while maintaining the high amplification of a shallow angle design. Mask accuracy does not affect kink actuators as much as it does chevron actuators because the kink part will have relatively larger angles while the rest of the beam remains horizontal. For example, the kink actuator shown in Figure 1(b) has a bend angle of 30 degrees yielding 46 raster steps in its bent length of $20 \mu \mathrm{m}$. Figure 2 clarifies the difference between both actuators.

\section{Design and Fabrication}

Chevron and kink actuators are two classes of the same bent-beam actuator morphology. Therefore, as shown in Figure 2, both can be defined using the same set of parameters: Arm Length (AL), Bent Length (BL), Bent Angle (BA), and Offset (OF). By applying a voltage between the anchor pads, the beams heat up and expand. Chevron actuators have angled beams that guide the buckling of the beams, whereas kink actuators have a kink in the middle that helps guide the motion. The defining difference between these two types of actuators is that chevron actuators consist primarily of bent length, whereas kink actuators consist primarily of a straight arm, which only has a small proportion of its length that is bent.

Several variations of kink and chevron actuators were fabricated using PolyMUMPS, a three-layer polysilicon surface micromachining process [13]. PolyMUMPS uses polysilicon as a structural material, with PSG (PhosphoSilicate Glass) as a sacrificial layer, and silicon nitride as electrical isolation between the polysilicon and the substrate [13].

\section{Experimental Procedure}

A total of 60 different actuator designs were tested, five different actuators of each design, in order to collect a statistically significant amount of data for both kink and chevron actuators. A probe station connected to an NI (National Instruments) control box was used to apply the voltage between the pads of the actuators. LABView software was written to output voltage to the probe station, to receive and process images from a camera mounted on the station, and to store the displacement results. The program was designed with various parameters, such as the range of input voltage, a measurement reference (a stationary mark on the substrate), defining the moving part of the actuator to be tracked and a scale for the captured images. The software processes the captured images and calculates the corresponding displacement for each voltage input. The data was collected incrementally: An actuator was first excited with a series of voltage steps ranging from $0 \mathrm{~V}$ to $3 \mathrm{~V}$, and the results at each voltage step were collected. This procedure was then repeated from $0 \mathrm{~V}$ to $4 \mathrm{~V}$ and so on until the actuator showed visible signs of deformation. The maximum voltage was determined for each actuator by examining the displacement data and recording the voltage at which the displacement started deviating 
from the previous data set. At this point the actuator was in the plastic deformation region and all subsequent displacement data was ignored since the actuator was no longer in its operating range. Most of the tested actuators entered their plastic deformation region around $10 \mathrm{~V}$, thus all of the data provided in this paper is up to $8 \mathrm{~V}$ as a safety margin. This process was repeated for the 60 different actuator designs.

\section{Actuator Simulation}

Simulations were carried out using COMSOL's Multiphysics software package. The thermal-electric-structural module was used to reproduce the displacement versus power graphs that were previously obtained in the experimental part. The polysilicon properties required for the simulation were obtained from various sources [14-18] and are summarized in Table 1. Because the ductile-brittle transition (DBT) temperature of polysilicon occurs at $925 \mathrm{~K}$ [14], all results with temperatures above this value were disregarded as the actuator would have entered the plastic deformation region. These results were verified by experimental data, and they were used to extract the stiffness values for both kink and chevron actuators for comparison. Figure 3 shows displacement results for both (a) a chevron actuator and (b) a kink actuator.

Table 1. Material properties used in running the COMSOL simulations.

\begin{tabular}{ccc}
\hline Property & Value & Reference \\
\hline Young's modulus of elasticity & $160 \mathrm{GPa}$ & {$[14]$} \\
Poisson's ratio & 0.22 & {$[14]$} \\
Thermal expansion coefficient & $3 \times 10^{-6}$ & {$[15,16]$} \\
Density & $2,330 \mathrm{~kg} / \mathrm{m}^{3}$ & {$[17]$} \\
Resistivity & $2.2 \times 10^{-5} \Omega \cdot \mathrm{m}$ & {$[16,18]$} \\
Resistance temperature coefficient & $3.17 \times 10^{-8} \Omega \cdot \mathrm{m} / \mathrm{K}$ & {$[18]$} \\
Thermal conductivity & $45 \mathrm{~W} /(\mathrm{m} \cdot \mathrm{K})$ & {$[18]$} \\
Heat capacity & $788.3 \mathrm{~J} /(\mathrm{kg} \cdot \mathrm{K})$ & {$[18]$} \\
Heat transfer coefficient & $11.1 \mathrm{~W} /\left(\mathrm{m}^{2} \cdot \mathrm{K}\right)$ & {$[18]$} \\
\hline
\end{tabular}

Figure 3. Comparison of the displacement $v s$. applied voltage graphs for both the experimental and simulation results for (a) a chevron (AL: 300, BL: 296, BA: 0.9, OF: 4.6), and (b) a kink (AL: 300, BL: 6, BA: 10, OF: 1.1).

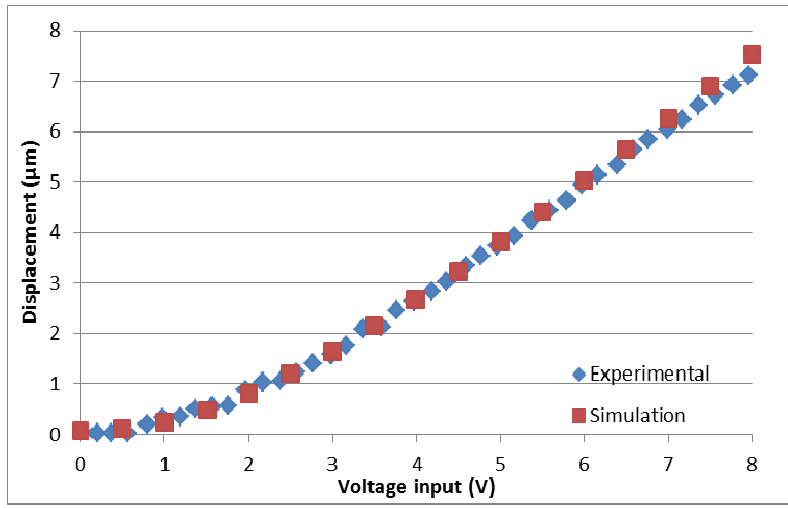

(a)

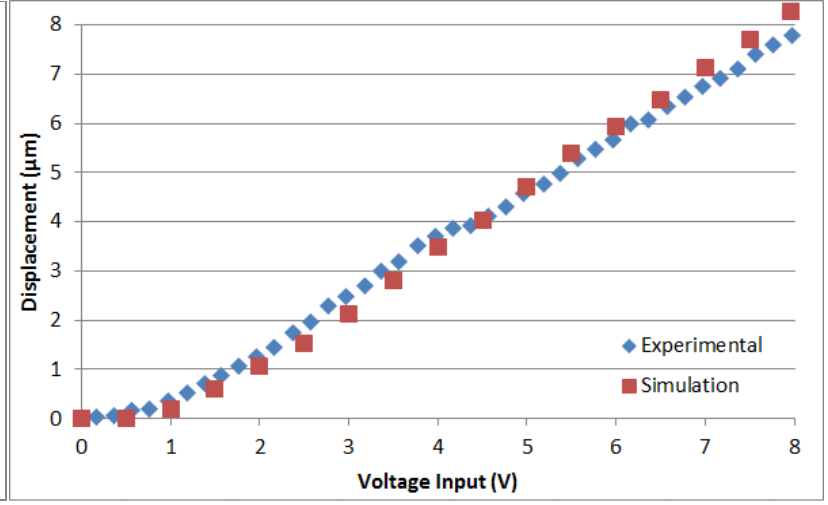

(b) 


\section{Results and Discussion}

The first parameter of interest is the displacement. Figure 4 shows the displacement vs. voltage graph for various chevron actuator designs with an Arm Length of $150 \mu \mathrm{m}$ and Bent Angles of 0.1-4.0 degrees. Figure 5 shows the same graph for kink actuators with an Arm Length of $150 \mu \mathrm{m}$ and varying Bent Lengths and Bent Angles. As shown in these figures, displacement values are higher for kink actuators of equivalent offsets at the same power input, which is mainly due to the fact that most of the displacement in the kink actuator is produced by the expansion of the horizontal zero angle beams.

Figure 4. Experimental displacement $v s$. applied voltage graph for chevron actuators with an arm length of $150 \mu \mathrm{m}$.

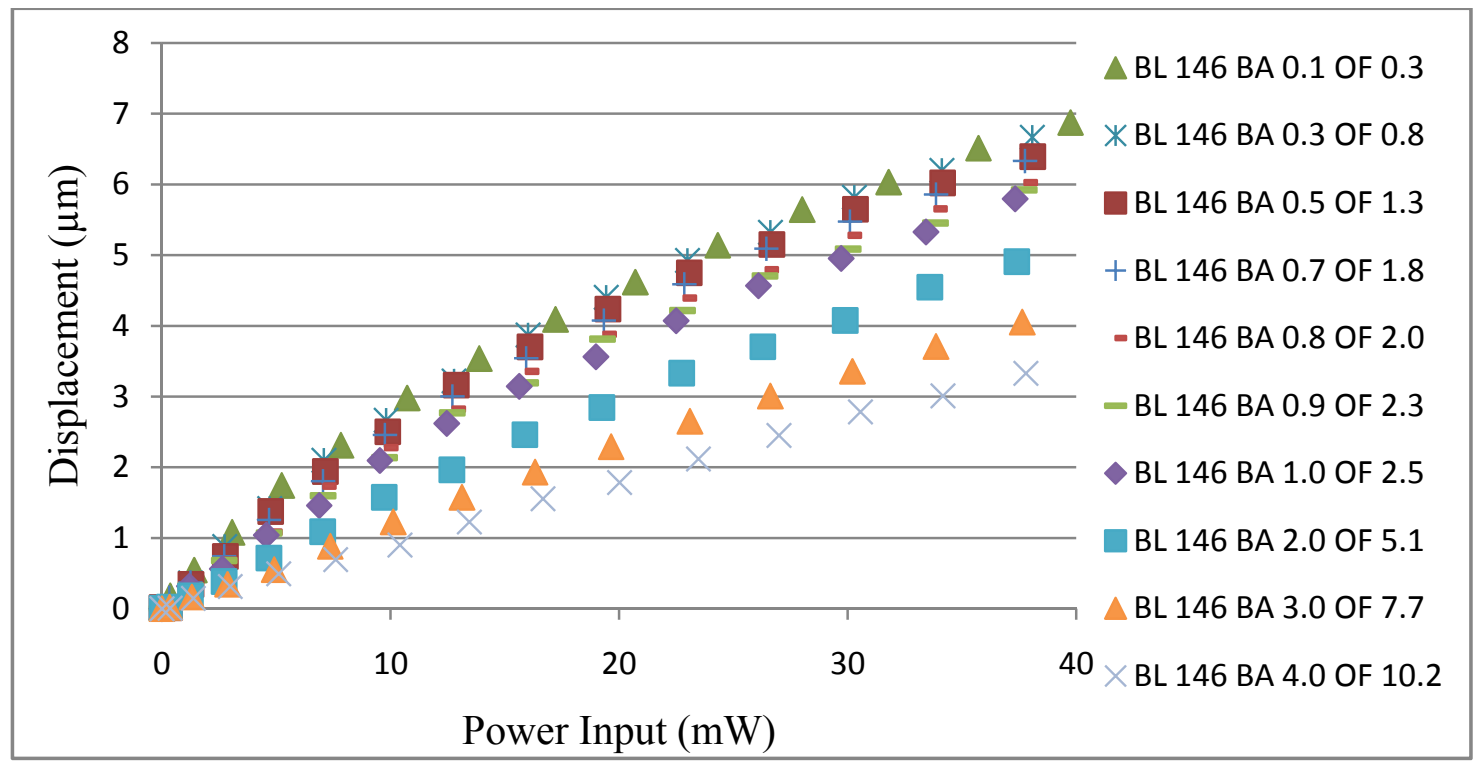

Figure 5. Experimental displacement $v s$. applied voltage graph of kink actuators with an arm length of $150 \mu \mathrm{m}$.

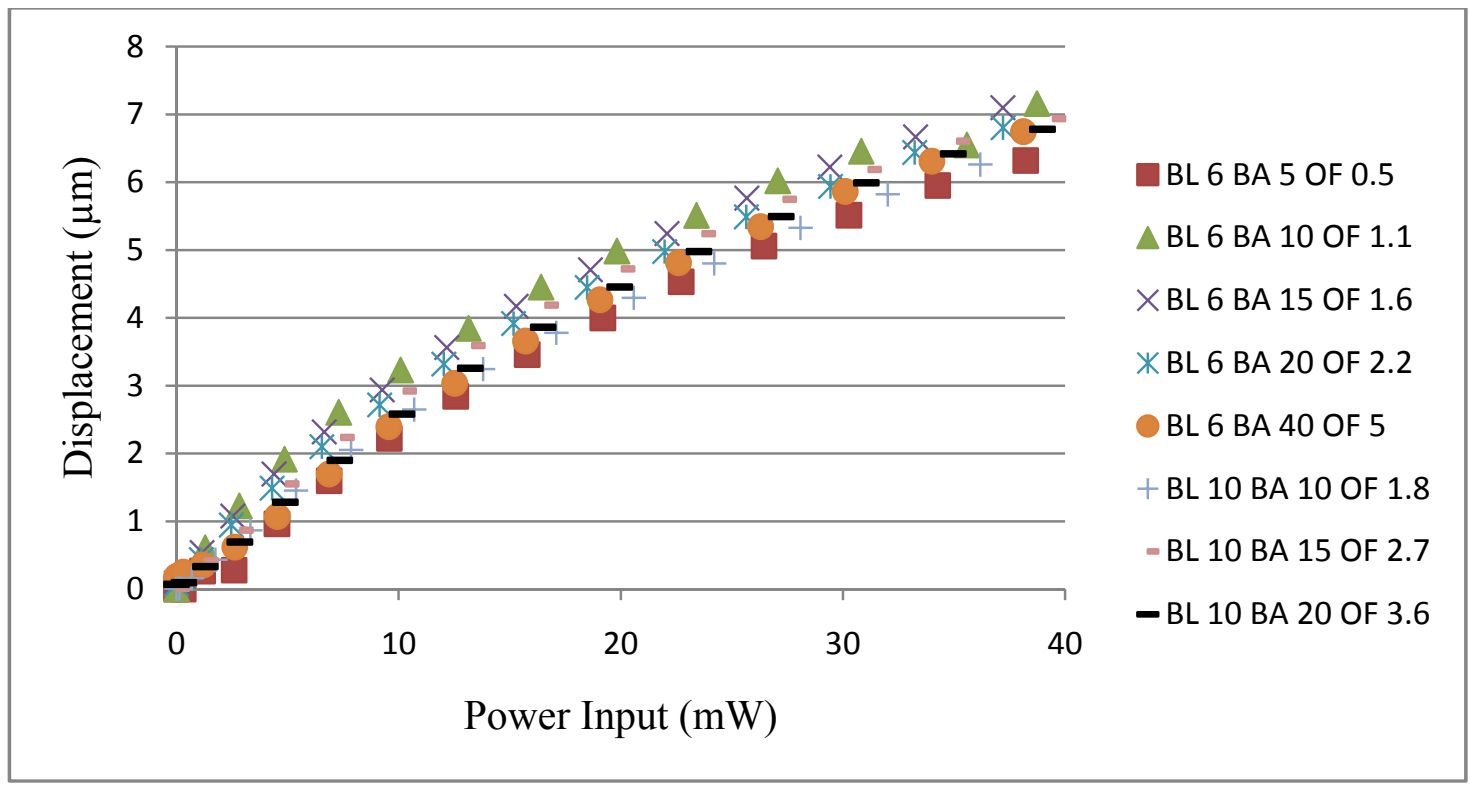


In Figures 4 and 5, the relationship between the displacement and the power input can be described as linear, and thus the slope of each line can be defined as the amplification factor (in $\mu \mathrm{m} / \mathrm{W}$ ). Figure 6 shows the amplification factors for the actuators plotted in Figures 4 and 5 versus the offset of the actuators. As shown in the figure, the amplification of chevron actuators increases with decreasing bend angle, which is directly related to the offset. However, as reported previously by Sinclair [3], at very small bend angles, the amplification factor begins to decrease because the actuators buckle out-of-plane instead of in-plane. The amplification factor for kink actuators is higher than the chevron actuators at all different offsets, as well as independent of the bend angle. This trend is seen over a wide range of bend angles for both chevrons and kink actuators.

Figure 6. Experimental amplification factor vs. their offsets for a set of chevron and kink actuators with $150 \mu \mathrm{m}$ arm lengths.

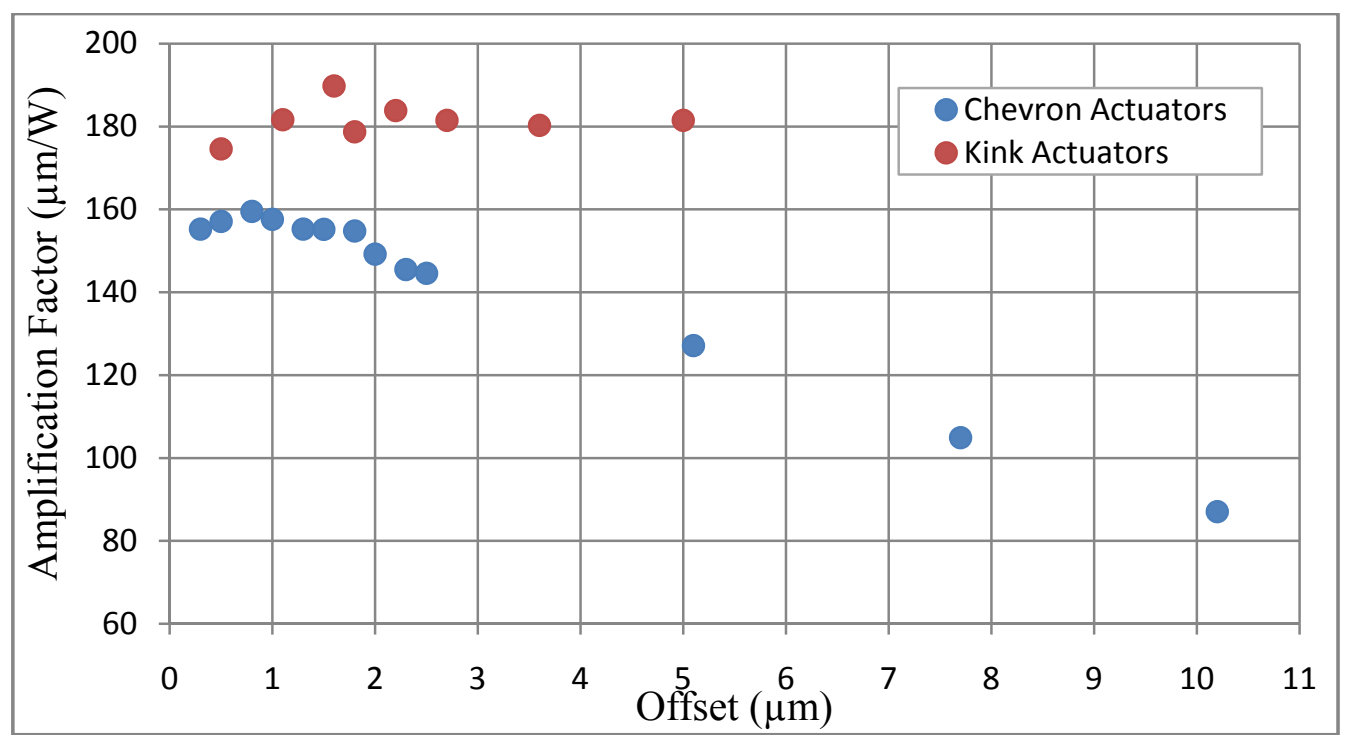

Figure 7. Comparison of the simulated stiffness $v s$. offset for chevron and kink actuators.

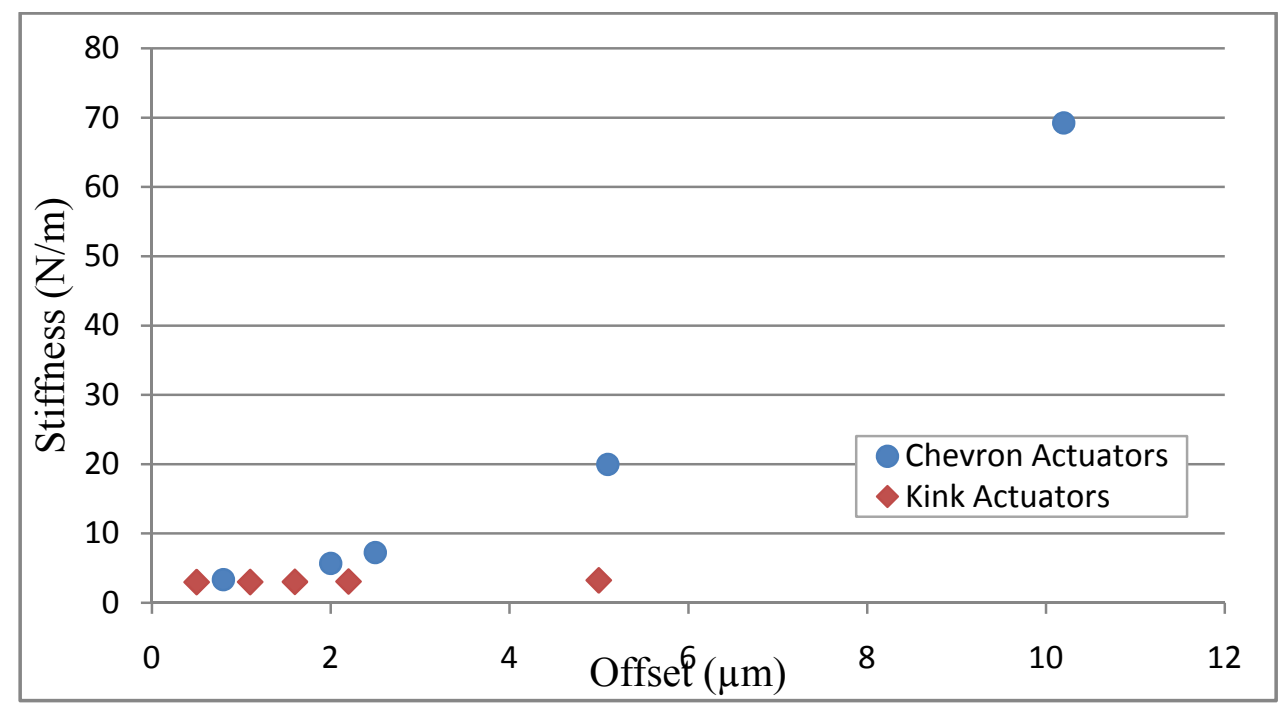

Stiffness is the ratio between the applied force and the maximum deflection occurring at the tip $[18,19]$. The stiffness of both types of actuator was determined using validated models and taking 
an unloaded actuator (i.e., no voltage input) and applying a force on the moving tip to extract the stiffness. Figure 7 shows the stiffness of chevron and kink actuators plotted against their offsets. For chevron actuators, stiffness increased with an increasing bend angle; however, the amplification factor was much lower after an offset of $2 \mu \mathrm{m}$, while kink actuators showed a constant stiffness for various values of offset. This result is expected because any kink actuator should have a stiffness close to the lowest bend angle chevron (due to the fact that kink beams are mostly horizontal with the exception of the middle part).

\section{Conclusion}

The work in this paper characterized the performance of a modified version of a bent-beam actuator, the kink actuator, and compared it to the standard chevron design. Various designs of kink and chevron actuators were fabricated out of polysilicon and then tested using a probe station. The displacement results were used to validate the thermal-electric-structural simulations produced by COMSOL. These simulations were then used to extract the stiffness of the actuators, which is not easily obtained experimentally.

The results show that kink actuators are capable of producing larger displacements at the same power input because most of the expansion is produced from the horizontal zero angle beams. Kink actuators also require lower power inputs compared to their chevron equivalents, which makes the kink actuator an excellent option where greater displacements are required. The stiffness for kink actuators is lower, but these actuators can be ganged together to produce larger forces, whereas the higher stiffness chevrons cannot provide large displacements.

The kink class of the bent-beam actuators demonstrates several advantages over the standard chevron shaped counterpart and should be considered for adoption into standard designs.

\section{References}

1. Cragun, R.; Howell, L.L. Linear thermomechanical microactuator. In Proccedings of the ASME International Mechanical Engineering Congress and Exposition, Microelectromechanical Systems (MEMS), Nashville, TN, USA, 14-19 November 1999; pp. 181-188.

2. Que, L.; Park, J.S.; Gianchandani, Y.B. Bent-beam electro-thermal actuators for high force applications. In Proccedings of the twelfth IEEE International Conference on Micro Electro Mechanical Systems, Orlando, FL, USA, 17-21 January 1999; pp. 31-36.

3. Sinclair, M.J. A high force low area MEMS thermal actuator. In Proccedings of the seventh Intersociety Conference on Thermal and Thermomechanical Phenomena in Electronic Systems, Las Vegas, NV, USA, 23-26 May 2000; p. 132.

4. Varona, J.; Tecpoyotl-Torres, M.; Hamoui, A.A. Design of MEMS vertical-horizontal chevron thermal actuators. Sens. Actuat. A-Phys. 2009, 153, 127-130.

5. Long, Q.; Jae-Sung, P.; Gianchandani, Y.B. Bent-beam electrothermal actuators-Part I: Single beam and cascaded devices. J. Microelectromechan. Syst. 2001, 10, 247-254.

6. Varona, J.; Tecpoyotl-Torres, M.; Escobedo-Alatorre, J.; Hamoui, A.A. Design and fabrication of a MEMS thermal actuator for 3D optical switching applications. In Proccedings of the Digest of the IEEE/LEOS Summer Topical Meetings, Acapulco, Mexico, 21-23 July 2008; pp. 31-32. 
7. Jae-Sung, P.; Chu, L.L.; Oliver, A.D.; Gianchandani, Y.B. Bent-beam electrothermal actuators-Part II: Linear and rotary microengines. J. Microelectromechan. Syst. 2001, 10, 255-262.

8. Gravier, S.; Coulombier, M.; Safi, A.; Andre, N.; Boe, A.; Raskin, J.P.; Pardoen, T. New on-chip nanomechanical testing laboratory-applications to aluminum and polysilicon thin films. J. Microelectromechan. Syst. 2009, 18, 555-569.

9. Daneshmand, M.; Fouladi, S.; Mansour, R.R.; Lisi, M.; Stajcer, T. Thermally actuated latching RF MEMS switch and its characteristics. IEEE Trans. Microwave Theory 2009, 57, 3229-3238.

10. Unamuno, A.; Uttamchandani, D. MEMS variable optical attenuator with vernier latching mechanism. IEEE Photonic Technol. Lett. 2006, 18, 88-90.

11. Wen-Chih, C.; Chia-Yu, W.; Chengkuo, L. Bi-directional movable latched micromechanism using one-directional movable chevron electrothermal actuators for switch and relay applications. In Proccedings of the Microprocesses and Nanotechnology Conference, Tokyo, Japan, 29-31 October 2003; pp. 180-181.

12. Guan, C.H.; Zhu, Y. An electrothermal microactuator with z-shaped beams. J. Micromechanic. Microengineer. 2010, doi: 10.1088/0960-1317/20/8/085014.

13. Carter, J.; Cowen, A.; Hardy, B.; Mahadevan, R.; Stonefield, M.; Wilcenski, S. Polymumps Design Handbook; MEMSCAP Inc.: Durham, NC, USA, 2006.

14. Rybicki, G.C.; Pirouz, P.; Aeronautics, U.S.N.; Scientific, S.A.; Division, T.I. Indentation Plasticity and Fracture in Silicon; National Aeronautics and Space Administration, Scientific and Technical Information Division: Washington, DC, USA, 1988.

15. Berry, B.S.; Pritchet, W.C. Stress and thermal expansion of boron doped silicon membranes on silicon substrates. J. Vac. Sci. Technol. A 1991, 9, 2231-2234.

16. Hickey, R.; Kujath, M.; Hubbard, T. Heat transfer analysis and optimization of two-beam microelectromechanical thermal actuators. J. Vac. Sci. Technol. A 2002, 20, 971-974.

17. Lide, D.R. Crc Handbook of Chemistry and Physics: A Ready-Reference Book of Chemical and Physical Data; CRC Press: Boca Raton, FL, USA, 2004.

18. Johnstone, R.W.; Parameswaran, M. Modelling surface-micromachined electrothermal actuators. Can. J. Elect. Comput. Eng. 2004, 29, 193-202.

19. Lai, Y.J.; McDonald, J.; Kujath, M.; Hubbard, T. Force, deflection and power measurements of toggled microthermal actuators. J. Micromechanic. Microengineer. 2004, 14, 49-56.

(C) 2012 by the authors; licensee MDPI, Basel, Switzerland. This article is an open access article distributed under the terms and conditions of the Creative Commons Attribution license (http://creativecommons.org/licenses/by/3.0/). 